\title{
Temporal Changes in Plasma Brain Natriuretic Peptide Levels During Exercise Stress-Echocardiography in Patients With Dilated Cardiomyopathy
}

\author{
Relationship to Left Ventricular Contractile Reserve
}

\author{
Vladan Peric, ${ }^{1}$ MD, Aleksandar Jovanovic, ${ }^{1}$ MD, Sasa Sovtic, ${ }^{1}$ MD, \\ Radojica Stolic, ${ }^{1}$ MD, Dijana Duikic, ${ }^{1} \mathrm{MD}$, and Petar Otasevic, ${ }^{2} \mathrm{MD}$
}

\begin{abstract}
SUMMARY
The aim of this study was to evaluate temporal changes in brain natriuretic petide (BNP) levels during exercise stress-echocardiography in patients with dilated cardiomyopathy with respect to the left ventricular contractile reserve. We studied 55 consecutive patients with dilated cardiomyopathy (mean age, $55 \pm 10$ years, 49 (89.1\%) male). All patients underwent exercise stress-echocardiography on a treadmill using the modified Bruce protocol. Contractile reserve was assessed by measuring changes in the wall motion score index ( $\triangle \mathrm{WMSI})$ at rest and and at peak exercise. Levels of BNP were measured at rest, in the first minute, and after 20 minutes following termination of the stress test. Thirty-six patients had preserved left ventricular contractile reserve and 19 patients did not. Patients with preserved left ventricular contractile reserve showed a continuous rise in BNP levels from baseline to peak exercise and to 20 minutes following exertion $(83.95 \pm 108.51$ versus $105.89 \pm 116.00$ versus $110.95 \pm 119.70 \mathrm{ng} / \mathrm{L}, P<0.001$, respectively). On the other hand, patients without preserved left ventricular contractile reserve showed a decline in BNP levels at peak exercise as compared to baseline $(335.49 \pm 693.11$ versus $320.08 \pm 562.60, P=0.031)$. $\Delta \mathrm{BNP}$ was positively correlated with preserved contractile reserve $(r=0.46, P=0.03)$ and lower NYHA class $(r=-0.65, P=0.001)$ in patients in whom baseline LVEF was lower than $20 \%$. Multivariate analysis identified only WMSI at rest (beta $-3.365, P=0.008,95 \%$ CI 0.03 to 0.411 ) as an independent predictor of left ventricular contractile reserve.

The increase in BNP levels during exercise stress-echocardiography is associated with preserved left ventricular contractile reserve in patients with dilated cardiomyopathy. (Int Heart J 2014; 55: 428-432)
\end{abstract}

Key words: Contractile reserve, Left ventricle, Treadmill

$\mathrm{D}$ ilated cardiomyopathy is associated with significant morbidity and mortality. A large number of variables have been examined as potential predictors of prognosis in these patients. ${ }^{1)}$ Left ventricular contractile reserve has been identified as one of the major prognostic indicators. Contractile reserve was most frequently assessed using pharmacological agents, such as dobutamine and dipyridamole, ${ }^{2,3)}$ but rarely using physical exertion. ${ }^{4)}$ It has been clearly demonstrated that preserved left ventricular contractile reserve was associated with milder forms of heart failure and identified patients with better prognosis. ${ }^{2-4)}$

The most important clinical use of BNP is to separate left ventricular dysfunction from other causes of dyspnea. ${ }^{5-7)}$ The diagnostic and prognostic value of BNP measurements at rest in patients with heart failure is very well documented, but the potential clinical implications of changes in BNP during physical exertion in these patients are much less clear.
There are initial reports indicating that the NT-pro BNP changes in response to dobutamine reflect improvement in left ventricular contractility and constitute an independent predictor of left ventricular inotropic reserve in patients with dilated cardiomyopathy, ${ }^{8)}$ and although resting NT-pro BNP levels can be used as a surrogate risk predictor, their changes during betaadrenergic stimulation showed no additive predictive value in assessing the clinical outcome during follow-up."

To the best of our knowledge, temporal changes in BNP levels during exercise stress echocardiography and the relation to left ventricular contractile reserve in patients with heart failure have not been examined. Therefore, we designed the present study to test the association of changes in BNP levels during exercise stress-echocardiography and the presence of left ventriclar contractile reserve in patients with dilated cardiomyopathy.

From the ${ }^{1}$ University of Pristina, School of Medicine, Internal Clinic, Kosovska Mitrovica and ${ }^{2}$ Dedinje Cardiovascular Institute, Belgrade University School of Medicine, Belgrade, Serbia.

Address for correspondence: Vladan Peric, MD, University of Pristina, School of Medicine, Kosovska Mitrovica, Anri Dinana bb, 38220 Kosovska Mitrovica, Serbia. E-mail: pericvladan@yahoo.com

Received for publication February 3, 2014. Revised and accepted February 21, 2014.

Released in advance online on J-STAGE August 4, 2014.

All rights reserved by the International Heart Journal Association. 


\section{METHODS}

Patients: We performed a prospective study at a tertiary cardiovascular center. The study included 55 consecutive patients with dilated cardiomyopathy who were treated in the outpatient clinic for heart failure. The average age was $55 \pm 10$ years, and $49(89.1 \%)$ of these patients were male. The diagnosis of dilated cardiomyopathy was based on echocardiographic findings and the findings of coronary angiography, if there were no data on the abuse of alcohol, malignant hypertension, exposure to toxins, myocarditis, hypertrophic cardiomyopathy, valvular heart disease, and/or significant coronary artery diseases (defined as $>50 \%$ of stenosis diameter of the major epicardial arteries). Coronary angiography was performed in all patients prior to the study. Standard heart failure therapy was used, including beta-blockers, ACE inhibitors, angiotensin receptor blockers, diuretics, aldosterone antagonists, digitalis, oral anticoagulants, and/or amiodarone. The study was approved by the local ethics committee.

Patients were included in the study if they met all of the following criteria: 1) age $>18$ years, 2) diagnosis of dilated cardiomyopathy, based on the above mentioned criteria; 3) a good echocardiographic window, defined as visualization of at least 13/17 segments, 4) left ventricular ejection fraction < $40 \%$, and 5) signed informed consent for participation in the study.

Criteria for exclusion were: 1 ) the inability to physically exert the patient, 2) complex ventricular arrhythmias at rest, and 3) NYHA class IV.

Exercise stress-echocardiography: Exercise tests were performed on a Cardiovit Schiller CS-200 treadmill (Schiller AG, Baar, Switzerland). All patients were subjected to symptoms limited exercise testing according to the modified Bruce protocol.

The test was discontinued earlier in case of complex ventricular arrhythmias. In all patients, beta-blockers were excluded from therapy two days prior to the test, while all other therapy was maintained. At all times during the test, a 12-lead ECG was recorded, and arterial blood pressure was measured at the end of each load level. During the test, the following variables were recorded: heart rate and the difference in heart rate at rest and at peak load, test duration, load level achieved, maximum workload (METs), double product, and adverse events.

All examinations were performed on a VIVID 4 echocardiograph (GEMS Ultrasound, Tirat Carmel, Israel) using a $3 \mathrm{~S}$ probe $(1.5-3.6 \mathrm{MHz})$ and recorded on hard disk for later analysis. All M-mode and $2 \mathrm{D}$ measurements were made according to the recommendations given by the American Society of Echocardiography. ${ }^{10)}$ Left ventricular volumes and the ejection fraction of the left ventricle (LVEF) were calculated based on a biplanar disc summation method (modified Simpson's rule). Regional wall motion of the left ventricle was analyzed on the basis of the division of the left ventricle into 17 segments, according to the recommendations of the American Society of Echocardiography. ${ }^{10)}$ Regional mobility has been denoted as: normal (scored as 1 point), hypokinesia - significantly reduced mobility ( 2 points), akinesia - the absence of systolic wall thickening ( 3 points), and dyskinesia - paradoxical movement of segments of the left ventricle in systole (4 points). The sum of the points obtained by scoring an individual segment is di- vided by number of scored segments and marked as the wall motion score index (WMSI). Segments that were not well visualized were not scored.

Echocardiographic images were recorded prior to the test and in the first minute following termination of the test. Left ventricular contractile reserve was determined using WMSI. Contractile reserve was defined as the difference between the WMSI in the first minute of the maximum load and its value in basal conditions $(\triangle \mathrm{WMSI})$. Based on the results of earlier studies it was assumed that the contractile reserve is preserved if $\Delta \mathrm{WMSI} \geq 0.19 .^{2)}$ Patients were divided into groups with preserved and non-preserved left ventricular contractile reserve.

BNP levels: Blood samples for BNP levels were taken from the median cubital vein of the non-dominant arm 30 minutes before the test, and within 1 minute and 20 minutes following the termination of the test. BNP levels were measured from all 3 blood samples for each patient. BNP levels were determined by chemiluminescence (Access 2 - Beckman Coulter, USA). Statistical analysis: All numerical parameters are expressed as the mean \pm standard deviation. The $t$-test for parametric numerical characteristics was used for examining the differences between the two defined groups and the Mann-Whitney test for numerical non-parametric features. To assess statistical differences in measured values of BNP baseline and during the test, Wilcoxon's matched pairs test and Friedman's test were used. Spearman's correlation was used to establish the correlation between changes in BNP and the examined variables. Univariate and multivariate analyses were performed to identify predictors of left ventricular contractile reserve.

\section{RESULTS}

A total of 36 patients were classified as having preserved left ventricular contractile reserve according to exercise induced changes in WMSI $\geq 0.19$, whereas in 19 patients contractile reserve was not preserved. The basic characteristics of the patients with and without preserved left ventricular contractile reserve are shown in Table I. There were no significant differences in age, gender, NYHA class, presence of atrial fibrillation, left bundle branch block, or the therapy that was used in these patients.

Patients with preserved left ventricular contractile reserve had smaller left ventricular end-diastolic $(62.95 \pm 5.64$ versus $68.58 \pm 7.53 \mathrm{~mm}, P=0.006)$ and end-systolic $(47.63 \pm 6.06$ versus $55.39 \pm 8.13 \mathrm{~mm}, P=0.001)$ diameters, a decreased left ventricular mass index $(159.68 \pm 31.90$ versus $197.06 \pm 54.04$ $\left.\mathrm{g} / \mathrm{m}^{2}, P=0.008\right)$, as well as a lower index of left ventricular volume at end-diastole $(70.94 \pm 24.45$ versus $94.92 \pm 33.13$ $\left.\mathrm{mL} / \mathrm{m}^{2}, P<0.001\right)$ and end-systole $(52.71 \pm 22.52$ versus $\left.78.71 \pm 33.04 \mathrm{~mL} / \mathrm{m}^{2}, P<0.001\right)$, higher left ventricular ejection fraction $(27.10 \pm 6.83 \%$ versus $18.67 \pm 7.28 \%, P<0.001)$, and a lower wall motion score index $(2.19 \pm 0.23$ versus $2.46 \pm$ $0.28, P=0.001)$ compared to the patients in whom contractile reserve was not preserved (Table II).

The basic characteristics of echocardiography stress-testing in patients with and without preserved left ventricular contractile reserve are shown in Supplemental Table. There were no significant differences in the parameters tested between the patients. No adverse events were observed during the test in either group. 
Table I. Baseline Demographic and Clinical Characteristics According to the Presence of Left Ventricular Contractile Reserve

\begin{tabular}{lccc}
\hline Variable & $\begin{array}{c}\Delta \text { WMSI } \geq 0.19 \\
(n=19)\end{array}$ & $\begin{array}{c}\Delta \text { WMSI }<0.19 \\
(n=36)\end{array}$ & $P$ \\
\hline Age & $55 \pm 13$ & $55 \pm 8$ & 0.54 \\
Gender (male) & $17(89.5 \%)$ & $32(88.9 \%)$ & 0.53 \\
Duration of illness (months) & $82 \pm 56$ & $77 \pm 45$ & 0.98 \\
NYHA class & $1.74 \pm 0.56$ & $1.83 \pm 0.70$ & 0.096 \\
Atrial fibrillation & $5(26.3 \%)$ & $6(16.7 \%)$ & 0.62 \\
LBBB & $4(21.1 \%)$ & $10(27.8 \%)$ & 0.75 \\
Therapy & & & \\
$\quad$ ACE inhibitor & $19(100 \%)$ & $34(94.4 \%)$ & 0.54 \\
Beta blocker & $18(94.7 \%)$ & $35(97.2 \%)$ & 0.64 \\
Diuretic & $18(94.7 \%)$ & $34(94.4 \%)$ & 0.96 \\
Spironolactone & $10(52.6 \%)$ & $20(55.6 \%)$ & 0.84 \\
Digitalis & $7(36.8 \%)$ & $17(47.2 \%)$ & 0.46 \\
Amiodarone & $3(15.8 \%)$ & $2(5.6 \%)$ & 0.33 \\
Anticoagulant & $6(31.6 \%)$ & $11(30.6 \%)$ & 0.94 \\
\hline
\end{tabular}

LBBB indicates left bundle branch block; NYHA, New York Heart Association Functional Classification; and WMSI, wall motion score index.

Table II. Echocardiographic Characteristics of Patients With and Without Preserved Left Ventricular Contractile Reserve

\begin{tabular}{lccc}
\hline Variable & $\Delta$ WMSI $\geq 0.19$ & $\Delta$ WMSI $<0.19$ & $P$ \\
\hline EDD $(\mathrm{mm})$ & $62.95 \pm 5.64$ & $68.58 \pm 7.53$ & 0.006 \\
ESD $(\mathrm{mm})$ & $47.63 \pm 6.08$ & $55.39 \pm 8.13$ & 0.001 \\
Left atrial diameter $(\mathrm{mm})$ & $42.21 \pm 5.44$ & $45.72 \pm 6.84$ & 0.058 \\
Left ventricular mass index $\left(\mathrm{g} / \mathrm{m}^{2}\right)$ & $159.68 \pm 31.90$ & $197.06 \pm 54.04$ & 0.008 \\
E/A ratio & $1.04 \pm 0.61$ & $0.96 \pm 0.59$ & 0.39 \\
E wave deceleration time $(\mathrm{ms})$ & $200.73 \pm 62.95$ & $173.70 \pm 56.14$ & 0.14 \\
Em medial annulus $(\mathrm{cm} / \mathrm{s})$ & $5.90 \pm 2.05$ & $5.19 \pm 2.19$ & 0.16 \\
E/Em ratio & $13.97 \pm 6.03$ & $15.85 \pm 7.39$ & 0.34 \\
EDV index (mL/m $\left.{ }^{2}\right)$ & $70.94 \pm 24.45$ & $94.92 \pm 33.13$ & $<0.001$ \\
ESV index (mL/m $\left.{ }^{2}\right)$ & $52.71 \pm 22.52$ & $78.71 \pm 33.04$ & $<0.001$ \\
Left ventricular ejection fraction $(\%)$ & $27.10 \pm 6.83$ & $18.67 \pm 7.28$ & $<0.001$ \\
Wall motion score index & $2.19 \pm 0.23$ & $2.46 \pm 0.28$ & 0.001 \\
\hline
\end{tabular}

EDD indicates left ventricular end-diastolic diameter; ESD, left ventricular end-systolic diameter; E, early left ventricular filling velocity; A, late left ventricular (atrial) filling velocity; Em, early mitral ring displacement; EDV, left ventricular volume at end diastole; and ESV, left ventricular volume at end systole.

Compared to the patients without preserved left ventricular contractile reserve, patients with preserved left ventricular contractile reserve had lower plasma concentrations of BNP at baseline $(83.95 \pm 108.51$ versus $335.49 \pm 693.11 \mathrm{ng} / \mathrm{L}, P=$ $0.017)$, at peak exercise $(105.89 \pm 116.00$ versus $320.08 \pm$ $562.60 \mathrm{ng} / \mathrm{L}, P=0.027)$ and 20 minutes after the test $(110.95$ \pm 119.70 versus $340.44 \pm 572.91 \mathrm{ng} / \mathrm{L}, P=0.046$ ) (Table III).

When examining temporal changes in BNP levels during echocardiography stress-testing, we found that patients with preserved left ventricular contractile reserve showed a continuous rise in BNP levels from baseline to peak exercise and to 20 minutes following exertion $(83.95 \pm 108.51$ versus $105.89 \pm$ 116.00 versus $110.95 \pm 119.70 \mathrm{ng} / \mathrm{L}, P<0.001$, respectively) (Figure). On the other hand, patients without preserved left ventricular contractile reserve showed a decline in BNP levels at peak exercise as compared to baseline $(335.49 \pm 693.11$ versus $320.08 \pm 562.60, P=0.031)$, but BNP values obtained after 20 minutes returned to the baseline values $(335.49 \pm 693.11$ versus $340.44 \pm 572.91 \mathrm{ng} / \mathrm{L}, P=\mathrm{NS}$ ) (Figure).

When patients were divided according to baseline LVEF (Table V), it became apparent that exercise induced changes in BNP significantly correlated with echocardiographic, clinical, and exercise parameters only in patients in whom baseline
LVEF was lower than $20 \%$. $\triangle \mathrm{BNP}$ in these patients was strongly correlated with preserved contractile reserve $(r=0.46$, $P=0.03)$, lower NYHA class $(r=-0.65, P=0.001)$, a larger increase in the double product $(r=0.49, P=0.02)$, work accomplished during the test $(r=0.48, P=0.02)$, and longer duration of the test $(r=0.51, P=0.01)$ (Table IV).

Univariate analysis identified WMSI at rest, ejection fraction at rest, and dichotomized change in BNP (ie, rise or fall from baseline to peak exercise) during exercise stress echocardiography as predictors of left ventricular contractile reserve. Conversely, age, sex, NYHA class, exercise duration, and baseline BNP values were not predictive of contractile reserve. Since WMSI at rest and ejection fraction at rest were highly correlated $(r=-0.887, P=0.0001)$, ejection fraction at rest was not included in multivariate analysis. Multivariate analysis identified only WMSI at rest (beta $-3.365, P=0.008,95 \% \mathrm{CI}$ 0.03 to 0.411 ) as an independent predictor of left ventricular contractile reserve.

\section{Discussion}

Previous studies mainly used dobutmaine or, less fre- 
Table III. Plasma BNP Levels in Patients With and Without Preserved Left Ventricular Contractile Reserve

\begin{tabular}{lrrr}
\hline Variable & $\Delta$ WMSI $\geq 0.19$ & $\Delta$ WMSI $<0.19$ & $P$ \\
\hline BNP at rest (ng/L) & $83.95 \pm 108.51$ & $335.49 \pm 693.11$ & 0.017 \\
BNP at peak exercise (ng/L) & $105.89 \pm 116.00$ & $320.08 \pm 562.60$ & 0.027 \\
BNP after 20 minutes (ng/L) & $110.95 \pm 119.70$ & $340.44 \pm 572.91$ & 0.046 \\
\hline
\end{tabular}

BNP indicates B-type natriuretic peptide; and $\triangle \mathrm{WMSI}$, changes in the wall motion score index.

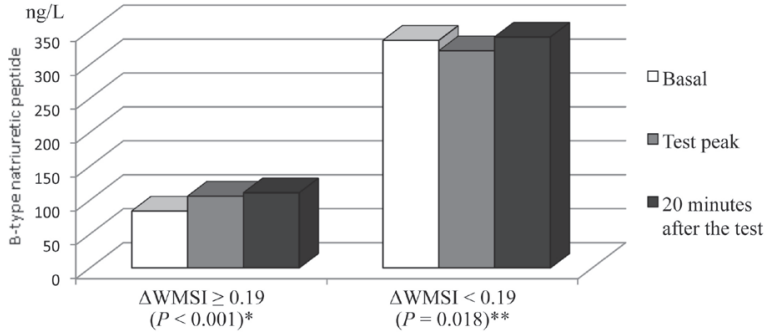

Figure. Temporal changes in B-type natriuretic peptide levels during stress echocardiography with respect to the presence of left ventricular contractile reserve $(* P<0.001$ to compare the values of basal and at the test peak, $P=0.083$ to compare the values of basal and 20 minutes after the test, $P=0.29$ to compare the values at the peak and 20 minutes after the test; $* * P=0.031$ to compare the values of basal and at the test peak, $P$ $=0.35$ to compare the values of basal and 20 minutes after the test, $P=$ 0.30 to compare the values at the peak and 20 minutes after the test). $\Delta$ WMSI indicates changes in the wall motion score index.

quently, adenosine stress echocardiographic protocols to test the presence of left ventricular contractile reserve in patients with left ventricular systolic dysfunction, whereas physical exertion, which is considered to be a truly physiologic inotropic stimulus, was rarely used. ${ }^{11,12}$ There is also considerable dispute over which index of left ventricular contractility to use, but changes in WMSI at maximum load as compared to baseline $(\Delta \mathrm{WMSI})$ emerged recently as the prognostically most important index with perspective for wider clinical use. ${ }^{2)}$

Our data indicate that patients in whom left ventricular contractile reserve was not preserved have lower ejection fraction, but greater left venticular dimensions, WMSI and mass than patients with preserved contractile reserve. These findings indicate that the myocardium of the patients without preserved left ventricular contractile reserve is significantly more damaged both structurally and functionally, which is consistent with previous findings. ${ }^{13)}$

On the other hand, there were no differences in the basic demographic variables, functional status, and therapy between the groups. Interestingly, duration of the test, achieved load, and other stress related variables were also similar. The reasons for this are not clear, but it may be assumed that these findings are due to the fact that our study included a small number of patients who were relatively oligosymptomatic, which did not allow for further stratification between the groups.

There is little data on temporal BNP changes during physical exertion in heart failure patients. Pascual-Figal and associates $^{14)}$ examined the release of BNP at peak exercise during symptom-limited exercise testing in patients with dilated cardiomyopathy. Their data indicated that patients with dilated cardiomyopathy may have a different pattern of BNP level change in response to physical exertion. The level of BNP increased during exercise testing in $63 \%$, did not change in
Table IV. Correlation Between Changes in Baseline and Peak Exercise BNP Levels and Clinical, Echocardiographic, and Exercise Variables According to Value of Left Ventricular Ejection Fraction

\begin{tabular}{lrrrr}
\hline Variable & \multicolumn{2}{c}{$\begin{array}{c}\mathrm{EF} \geq 20 \% \\
(n=24)\end{array}$} & \multicolumn{2}{c}{$\begin{array}{c}\mathrm{EF}<20 \% \\
(n=31)\end{array}$} \\
& $r$ & $P$ & $r$ & $P$ \\
\hline$\Delta$ WMSI & -0.02 & 0.92 & 0.46 & 0.03 \\
NYHA class & 0.15 & 0.43 & -0.65 & 0.001 \\
Difference in double product & 0.11 & 0.56 & 0.49 & 0.02 \\
Achieved METs & 0.28 & 0.12 & 0.48 & 0.02 \\
Duration of test & 0.21 & 0.26 & 0.51 & 0.01 \\
\hline
\end{tabular}

BNP indicates B-type natriuretic peptide; MET, metabolic equivalent; NYHA, New York Heart Association; and $\Delta$ WMSI, changes in the wall motion score index.

$22 \%$, and decreased in $15 \%$ of the patients. Baseline plasma concentration of BNP and rise in BNP during the test were inversely correlated. Age and a low ejection fraction were identified as independent predictors of higher levels of BNP at rest, as was a lower BNP response to exrecise. BNP at rest was negatively, and BNP increase during exercise testing positively correlated with functional capacity.

Our data were similar to the results of a previous study, suggesting that a BNP increase during physical exertion may identify patients with better functional capacity and less structural and functional damage of the myocardium. Furthermore, we extend the findings of the previous study by: 1) linking changes in BNP levels during exercise to the presence of left ventricular contractile reserve, and 2) adding additional sampling of BNP levels 20 minutes following exercise termination. Previous studies have shown that the release of BNP from secretory granules occurs in the first few minutes of physical exertion ${ }^{15)}$ and that the highest levels of BNP may be recorded precisely at the moment of the maximum load achieved. ${ }^{16)} \mathrm{We}$ showed that there is a continuous increase in BNP levels 20 minutes after exercise termination in patients with preserved left ventricular contractile reserve. The potential clinical and prognostic implications of these findings are not clear at the moment.

The decrease in BNP level during physical exertion in patients without preserved left ventricular contractile reserve is probably due to reduced production or release. Patients without preserved left ventricular contractile reserve have functionally and structurally damaged myocardium, whose secretory capacity may be reduced, so it can not respond to emerging circulatory demands that occur during exertion, due to a relative lack or exhaustion of BNP. ${ }^{17)}$ On the other hand, failure of further ANP release was reported in patients with a pronounced chronic increase in atrial volume and pressure, and it can be hypothetized that the same may apply for BNP secretion from the ventricles. ${ }^{18)}$ In some patients, the reason for the 
decrease in the level of BNP during physical activity may be its increased clearance, due to its increased degradation by neutral endopeptidase, which is mostly present in the proximal tubules of the kidney. ${ }^{19)}$ Improved blood flow through the kidneys favors this phenomenon.

Studies which pharmacologically examined left ventricular contractile reserve showed different results. Parthenakis and coworkers ${ }^{20)}$ examined the change of NT pro-BNP 60 minutes following low-dose dobutamine stress echocardiographic testing and showed that the reduction of BNP concentration following the test was an independent predictor of preserved left ventricular contractile reserve. These findings were explained by the acute hemodynamic improvement achieved by lowdose dobutamine in patients with preserved contractile reserve, leading in turn to the decrese in BNP levels. Dobutamine, unlike physical exertion, first increases myocardial contractility via activation of beta receptors with only a minimal increase in heart rate. ${ }^{21)}$ In patients with chronic heart failure, dobutamine also reduces systemic vascular resistance and thereby increases cardiac output with little change in arterial blood pressure. On the other hand, physical exertion causes an increase in heart rate from the very beginning, followed by increases in blood pressure and in circulating volume.

It has been shown recently that changes in NT-pro BNP levels during low dose dobutamine $(5-15 \mu \mathrm{g} / \mathrm{kg} /$ minute) stress echocardiography have no additive prognostic value in the assessment of clinical outcome during long-term follow-up. ${ }^{9)} \mathrm{Fu}-$ ture studies are needed to assess the potential prognostic implications of temporal BNP changes during exercise stress echocardiography.

The present study has a number of limitations. The small number of patients did not allow for further subgroup analysis. No hemodynamic or cardiopulmonary measurements were made, so it was not possible to study the relationship between hemodynamic parameters or oxygen consumption and changes in BNP levels.

In conclusion, the increase in BNP levels during exercise stress-echocardiography is associated with preserved left ventricular contractile reserve in patients with dilated cardiomyopathy.

\section{REFERENCES}

1. Bahler RC. Assessment of prognosis in idiopathic dilated cardiomyopathy. Chest 2002; 121: 1016-9.

2. Otasevic P, Popovic ZB, Vasiljevic JD, et al. Head-to-head comparison of indices of left ventricular contractile reserve assessed by high-dose dobutamine stress echocardiography in idiopathic dilated cardiomyopathy: five-year follow up. Heart 2006; 92: 1253-8.

3. Pratali L, Otasevic P, Neskovic A, Molinaro S, Picano E. Prognostic value of pharmacologic stress echocardiography in patients with idiopathic dilated cardiomyopathy: a prospective, head-tohead comparison between dipyridamole and dobutamine test. J Card Fail 2007; 13: 836-42.

4. Latham RD, Thornton JW, Mulrow JP. Cardiovascular reserve in idiopathic dilated cardiomyopathy as determined by exercise response during cardiac catheterization. Am J Cardiol 1987; 59:
1375-9.

5. Friedewald VE Jr, Burnett JC Jr, Januzzi JL Jr, Roberts WC, Yancy $\mathrm{CW}$. The editor's roundtable: B-type natriuretic peptide. Am J Cardiol 2008; 101: 1733-40. (Review)

6. Maisel AS, Krishnaswamy P, Nowak RM, et al. Rapid measurement of B-type natriuretic peptide in the emergency diagnosis of heart failure. N Engl J Med 2002; 347: 161-7.

7. Koglin J, Pehlibanti S, Schwaiblmair M, Vogeser M, Cremer P, von Scheidt W. Role of brain natriuretic peptide in risk stratification of patients with congestive heart failure. J Am Coll Cardiol 2001; 38: 1934-41

8. Parthenakis FI, Patrianakos AP, Haritakis CN, Zacharis EA, Nyktari EG, Vardas PE. NT-proBNP response to dobutamine stress echocardiography predicts left ventricular contractile reserve in dilated cardiomyopathy. Eur J Heart Fail 2008; 10: 475-81.

9. Parthenakis F, Patrianakos A, Nyktari E, Arfanakis D, Zacharis E, Vardas P. Prognostic value of NT-pro BNP, left ventricular inotropic reserve and cardiopulmonary exercise test in patients with non-ischemic dilated cardiomyopathy. Int J Cardiol 2011; 147: 326-8.

10. Gardin JM, Adams DB, Douglas PS, et al. Recommendations for a standardized report for adult transthoracic echocardiography: a report from the American Society of Echocardiography's Nomenclature and Standards Committee and Task Force for a Standardized Echocardiography Report. J Am Soc Echocardiogr 2002; 15: 275-90.

11. Pratali L, Otasevic P, Rigo F, Gherardi S, Neskovic A, Picano E. The additive prognostic value of restrictive pattern and dipyridamole-induced contractile reserve in idiopathic dilated cardiomyopathy. Eur J Heart Fail 2005; 7: 844-51.

12. Nagaoka H, Isobe $\mathrm{N}$, Kubota $\mathrm{S}$, et al. Myocardial contractile reserve as prognostic determinant in patients with idiopathic dilated cardiomyopathy without overt heart failure. Chest 1997; 111: 34450.

13. Schinkel AF, Vourvouri EC, Bax JJ, et al. Relation between left ventricular contractile reserve during low dose dobutamine echocardiography and plasma concentrations of natriuretic peptides. Heart 2004; 90: 293-6.

14. Pascual-Figal DA, Peñafiel P, de la Morena G, et al. Relation of B-type natriuretic peptide levels before and after exercise and functional capacity in patients with idiopathic dilated cardiomyopathy. Am J Cardiol 2007; 99: 1279-83.

15. de Bold AJ, Bruneau BG, Kuroski de Bold ML. Mechanical and neuroendocrine regulation of the endocrine heart. Cardiovasc Res 1996; 31: 7-18. (Review)

16. Steele IC, McDowell G, Moore A, et al. Responses of atrial natriuretic peptide and brain natriuretic peptide to exercise in patients with chronic heart failure and normal control subjects. Eur J Clin Invest 1997; 27: 270-6.

17. Krüger $\mathrm{S}$, Graf J, Merx MW, et al. Brain natriuretic peptide kinetics during dynamic exercise in patients with chronic heart failure. Int J Cardiol 2004; 95: 49-54.

18. Redfield MM, Edwards BS, McGoon MD, Heublein DM, Aarhus LL, Burnett JC Jr. Failure of atrial natriuretic factor to increase with volume expansion in acute and chronic congestive heart failure in the dog. Circulation 1989; 80: 651-7.

19. Kenny AJ, Stephenson SL. Role of endopeptidase-24.11 in the inactivation of atrial natriuretic peptide. FEBS Lett 1988; 232: 1-8. (Review)

20. Parthenakis FI, Patrianakos AP, Haritakis CN, Zacharis EA, Nyktari EG, Vardas PE. NT-proBNP response to dobutamine stress echocardiography predicts left ventricular contractile reserve in dilated cardiomyopathy. Eur J Heart Fail 2008; 10: 475-81.

21. Leier CV, Unverrfeth DV. Drugs five years later. Dobutamine. Am J Med 1983; 99: 490-6. 